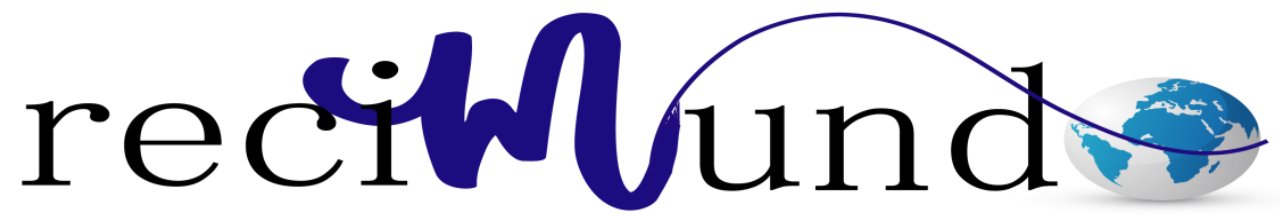

Revista Científica Mundo de la Investigación y el Conocimiento

Vintimilla Burgos Napoleón Patricio a; Plúas Robles Cecilia Isabel ${ }^{\text {b; }}$ Piña Tornés

Arlines A ${ }^{\text {c; }}$ Lenin Stalin Suasnabas Pacheco ${ }^{\text {d }}$

Mentoplastia medio terapéutico y estético

Revista Científica Mundo de la Investigación y el Conocimiento. Vol. 1 núm., 5, diciembre, 2017, pp. 814-830

DOI: $10.26820 /$ recimundo/1.5.2017.814-830

Editorial Saberes del Conocimiento

a. Universidad Guayaquil - Facultad de Odontología; Docente a Tiempo Completo; Especialista en Cirugía Plástica, Reconstructiva y Estética; napoleon.vintimillab@ug.edu.ec

b. Universidad Guayaquil - Facultad de Odontología; Docente a Tiempo Completo; Especialista en Cirugía Plástica, Reconstructiva y Estética; drapluas@ hotmail.com

c. Universidad Guayaquil - Facultad de Odontología; Docente a Tiempo Completo; Especialista en Cirugía Plástica, Reconstructiva y Estética; arlines.pinat@ug.edu.ec

d. Universidad Guayaquil- Facultad de Odontología; Docente a Tiempo Completo; Magister en Gerencia de Tecnologías de la Información; lenin.suasnabas@ug.edu.ec 


\section{Mentoplastia medio terapéutico y estético}

Vol. 1, núm. 5., (2017)

Vintimilla Burgos Napoleón Patricio; Plúas Robles Cecilia Isabel; Piña Tornés Arlines A.;

Lenin Stalin Suasnabas Pacheco

\section{Resumen}

El mentón es una de las zonas faciales más visibles, situadas a nivel inferior y medial de nuestro rostro, en la región mentoniana; cumple un papel importante en el contorno facial ya que si este es muy pronunciado hace que una persona delgada se vea un poco más robusta.

La mentoplastía es un acto quirúrgico que consiste en realizar modificaciones en el mentón tanto en altura o forma corrigiendo así deformaciones y la estética de un paciente. Esté procedimiento se puede realizar solo o combinada con otras cirugías estéticas como la rinoplastia si se quiere llegar a una perfiloplastía, asimismo es posible asociarla a cirugía ortognática.

En la actualidad se han realizado estudios que evidencian que el mentón también produce lo que son los ronquidos es por ello que esta cirugía es realizada no solo por estética sino por una anomalía fisiológica de la persona.

Palabras clave: Mentoplastía. Perfiloplastia. Cirugía Ortognática. Mentón. 


\section{Mentoplastia medio terapéutico y estético}

Vol. 1, núm. 5., (2017)

Vintimilla Burgos Napoleón Patricio; Plúas Robles Cecilia Isabel; Piña Tornés Arlines A.; Lenin Stalin Suasnabas Pacheco

\section{Summary}

The chin is one of the most visible facial areas, located below and medial level of our face, in the chin area; plays an important role in facial contours as if it is pronounced is a thin person makes it look a little more robust.

The mentoplasty is a surgical procedure that involves making changes to the chin in height or shape deformations and thus correcting the aesthetics of a patient. Be procedure can be performed alone or combined with other cosmetic surgeries like rhinoplasty if you want to reach a Perfiloplastia also may associate with orthognathic surgery.

At present there have been studies of the chin also produces what are snoring is why this surgery is performed not only for aesthetics but for a physiological abnormality of the person.

Keywords: Mentoplasty. Profileoplasty. Orthognathic surgery. Chin. 


\section{Mentoplastia medio terapéutico y estético}

Vol. 1, núm. 5., (2017)

Vintimilla Burgos Napoleón Patricio; Plúas Robles Cecilia Isabel; Piña Tornés Arlines A.;

Lenin Stalin Suasnabas Pacheco

\section{Introducción.}

El mentón es una estructura facial ubicada en la cara en una zona muy visible de nuestro perfil. Esta es la causa por la que debemos corregir sus malformaciones; y de utilizarlo como medio terapéutico para el tratamiento de otras anomalías dentofaciales, ya que nos sirve como hueso para implante óseo autólogo (implantología). Según Laura García, Arturo Bilbao y Juan Grau podemos utilizar como injerto óseo autólogo intraoral a la sínfisis (mentón) y ramas ascendentes mandibulares, pudiendo tomar también hueso de la tuberosidad maxilar, la apófisis coronoides, el arbotante máxilo-malar o los torus.(Injertos óseos en Implantología, pág. 151)

El mentón al servir como injerto óseo nos sirve para corregir malformaciones dentofaciales y no solo como estética. Según (Valdivieso, 2012)“La Cirugía Plástica a nuestra forma de ver es siempre reconstructiva",

La cirugía plástica permite hacer esos cambios de inconformidad de la persona, hoy en día la mentoplastía es muy solicitada por las personas que han perdido peso o no se siente bien y desean realizarse una reconstrucción del mentón.

Al hablar de estética tenemos claro que es un proceso en el cual mejoramos algo o le damos otra perspectiva, exactamente de esto se trata, ayudar a corregir al mentón dándole una nueva imagen o a su vez mejorando lo que ya está definido mediante terapias para regular este proceso.

No siempre es fácil la corrección del mentón pero con una cirugía o un tratamiento adecuado podremos obtener el resultado esperado y esto lo vamos a demostrar. 


\section{Mentoplastia medio terapéutico y estético}

Vol. 1, núm. 5., (2017)

Vintimilla Burgos Napoleón Patricio; Plúas Robles Cecilia Isabel; Piña Tornés Arlines A.; Lenin Stalin Suasnabas Pacheco

\section{Metodología.}

La metodología que se utilizó para este tema investigativo, se inserta en un estudio bibliográfico de tipo documental y de carácter descriptivo.

De acuerdo con (Arias, 2006) "El nivel de investigación se refiere al grado de profundidad con que se aborda un objeto o fenómeno, y este puede ser exploratorio, descriptivo o explicativo".

Para la realización de este trabajo se requiere de una definición clara y concisa de cada uno de los aportes y necesidades de una documentación; que permite el sustento y veracidad del artículo elaborado y obtener nuevos conocimientos para el estudio del mismo.

La técnica que se ha utilizado es a través de las fichas bibliográficas.

Los datos secundarios según (Sabino, 1986) "Son registros escritos que proceden también de un contacto con la práctica, pero que ya han sido recogidos y procesados por otros investigadores". En este trabajo los datos secundarios están representados por los antecedentes de estudio, los textos que han sido base para la elaboración de este documento, las teorías que sustentan el proceso aquí descritos y todo apoyo teórico presente en esta investigación entra dentro de esta clasificación.

Las categorías consideradas por los autores fueron:

a) Definición de Mentoplastía.

b) Análisis de malformaciones del mentón. 


\section{Mentoplastia medio terapéutico y estético}

Vol. 1, núm. 5., (2017)

Vintimilla Burgos Napoleón Patricio; Plúas Robles Cecilia Isabel; Piña Tornés Arlines A.;

Lenin Stalin Suasnabas Pacheco
c) Diagnóstico
d) Técnica quirúrgica.
e) Complicaciones y riesgos.
f) Discusión.
g) Conclusiones.

\section{Definición de Mentoplastía}

Según (Gilbert, 2013) La mentoplastía es también conocida como genioplastia, es un procedimiento quirúrgico que busca mediante diversos medios lograr un aumento en la proyección del mentón”' (2013)

El mentón forma una estructura importante de los rasgos faciales, estos al ser muy pronunciados logra hacer ver a la persona de una cara ancha, cuando el mentón es pequeño o reducido en un individuo de rasgos faciales anchos el mentón logra perderse y se pronuncia en la persona la papada.

El mentón es una de las zonas faciales más visibles, y como lo indica el Dr. Morera es un complejo donde cualquier condición y modificación alterará y cambiará este conjunto.

"Según el Dr. Eduardo Morera Serna, anatómicamente el mentón se define como el área comprendida debajo del pliegue labiomental. Cuando observamos el rostro desde una visión frontal, es difícil distinguir el mentón del labio inferior, por lo que se evalúa en conjunto el complejo labio inferior-surco labiomental-mentón- 


\section{Mentoplastia medio terapéutico y estético}

Vol. 1, núm. 5., (2017)

Vintimilla Burgos Napoleón Patricio; Plúas Robles Cecilia Isabel; Piña Tornés Arlines A.; Lenin Stalin Suasnabas Pacheco

región submentocervical. Cualquier procedimiento que busque alterar el mentón modificará este conjunto de estructuras al completo(Morera, pág. 350)”

Es por ello que al realizar una modificación en el mentón se modifican sus planos constitutivos; como lo es el hueso maxilar, la piel, músculos, periostio y sobretodo el cuidado que se debe tener por los vasos y nervios que se encuentran en esta región.

Según L. Testut; O. Jacob la región mentoniana es como dos precedentes, impar y media. Comprende la eminencia mentoniana del maxilar inferior y partes blandas que por delante la cubren. (Tratado de Anatomia Topográfica, pág. 221)

En la actualidad las personas buscan mejorar estéticamente y sentirse bien con su imagen y por ende consigo mismos.

Según (Pazmiño, 2014) 'La mentoplastía es mejorar la proporción de los rasgos faciales el cual puede ser el aumento o la disminución del mentón'”.

Según (Mollinedo Patzi, 2011) “La mentoplastía es un procedimiento mediante el cual se busca lograr un aumento o reducción en la proyección del mentón o corregir alteraciones en su forma, a través de medios quirúrgicos, con el único fin de conseguir estética y armonía del rostro, ya que el mentón o barbilla es uno de los elementos más resaltantes de la cara e influye decisivamente en el equilibrio facial." 


\section{Mentoplastia medio terapéutico y estético}

Vol. 1, núm. 5., (2017)

Vintimilla Burgos Napoleón Patricio; Plúas Robles Cecilia Isabel; Piña Tornés Arlines A.; Lenin Stalin Suasnabas Pacheco

Gracias a la cirugía podemos obtener un mejor rostro; aunque a la mayoría no le importe si corre riesgo a través de ello. En todo el mundo está de moda lo que es con la cirugía estética, hoy en día se realiza mucho lo que es la mentoplastía o la reducción del mentón.

Según (Macías, 2013) “Las intervenciones para el aumento o disminución del mentón se ha incrementado en los últimos años en nuestro país’”

En la actualidad ha aumentado el índice de cirugías plásticas porque el ser humano busca la perfección y con ello sentirse bien, la mentoplastía en la actualidad se realiza mucho en las personas que han perdido peso y no se siente conforme con su aspecto facial debido a los cambios en su cuerpo.

Según (Ruiz, 2014)“A las mujeres del otro lado del Atlántico ya no les interesa tanto aumentar su talla del sujetador, reducir 'sus cartucheras' o aplicarse Bótox. Lo que está de moda ahora entre ellas, aunque también entre ellos, es el aumento del mentón o barbilla (mentoplastía), a tenor de un informe que acaba de emitir la Sociedad Americana de Cirujanos Plásticos (ASPS, sus siglas en inglés). El organismo establece un incremento total del $71 \%$ de este tipo de intervenciones en 2011."

El mentón juega un papel importante en el contorno facial ya que este si es muy pronunciado hace que una persona delgada se vea un poco más robusta.

Según (Solano, 2013) “El mentón con la frente y la nariz juega un papel importante en la armonía facial"’ 


\section{Mentoplastia medio terapéutico y estético}

Vol. 1, núm. 5., (2017)

Vintimilla Burgos Napoleón Patricio; Plúas Robles Cecilia Isabel; Piña Tornés Arlines A.; Lenin Stalin Suasnabas Pacheco

Hoy en día se realizan muchas cirugías plásticas para mejorar el rostro; muchas personas piensan que a través de ello se puede obtener beneficios, ya que esta cirugía les pueden abrir muchas puertas, pero debemos tener conciencia de que esta también puede presentar complicaciones.

\section{Análisis de Malformaciones del mentón}

Para poder realizar una mentoplastía debemos saber que vamos a corregir es por ello que debemos hablar de las deformaciones del mentón.Como la micrognatia que es la falta de desarrollo del mentón y la prognatia que es el desarrollo excesivo del mentón.

Según Fernando Ramírez y Alejandra Buñuelos las deformaciones de mentón son: La microgenia es la más frecuente de todas y el desarrollo insuficiente está limitado a la porción anterior (sínfisis mentoniana), lo cual origina mandíbula en retrusión, pero sin maloclusión. La falta de desarrollo puede ser en sentido vertical, anteroposterior o mixto. La retrognatia se distingue por retrusión de la mandíbula de tamaño relativamente normal, acompañado de maloclusión dental.(pág. 65)

Las deformaciones del mentón como la microgeniamicrognatia y la retrognatia no solo causan un problema estético y funcional si no psicológico privándole a la persona en su buen vivir. 


\section{Mentoplastia medio terapéutico y estético}

Vol. 1, núm. 5., (2017)

Vintimilla Burgos Napoleón Patricio; Plúas Robles Cecilia Isabel; Piña Tornés Arlines A.; Lenin Stalin Suasnabas Pacheco

Definición de mentoplastía o cirugía de mentón

Según (Mollinedo Marcela, 2014) “La mentoplastía es un procedimiento quirúrgico cuyo objetivo es realizar cambios en el aspecto del mentón, dichas modificaciones se llevan a cabo en diferentes planos del espacio, pues con esta cirugía estética se logra la reducción o aumento de la proyección del mentón, cambio en su altura o bien una mejoría en su forma."(Mentoplastia , pág. 5)

La mentoplastía es un acto quirúrgico que consiste en realizar modificaciones en el mentón tanto en altura o forma corrigiendo así deformaciones y la estética de un paciente.

Según (Burgoa, 2012) “La mentoplastía consiste en la reconstrucción, modificación, reducción o ampliación del mentón, mediante la aplicación de prótesis, misma que se utiliza en el primer y último caso, mientras que la reducción se relaciona a la osteotomía."

No solo se puede realizar una ampliación del mentón, si no que para las personas que tienen algún defecto con su mentón, también pueden modificar o reconstruir. Para tener un aspecto mejor.

Según(Gacitúa Garstman, 2012) “La cirugía de mentón puede combinarse con otras cirugías plásticas faciales, como la cirugía de nariz y la de papada, para afinar el rostro y darle mayor armonía." 


\section{Mentoplastia medio terapéutico y estético}

Vol. 1, núm. 5., (2017)

Vintimilla Burgos Napoleón Patricio; Plúas Robles Cecilia Isabel; Piña Tornés Arlines A.; Lenin Stalin Suasnabas Pacheco

Para mucha gente hoy en día la cirugía se ha vuelto indispensable; ya que por eso podemos cambiar algunos aspectos, que no estemos a gusto con nuestro cuerpo.

Según (Freschi, 2011) “Hacerse una cirugía plástica de cualquier tipo tanto, de implante de mentón como implante de mama, etc. es una decisión de suma importancia y nunca debe ser apresurada, todo lo contario, debe ser fruto de un profundo análisis y reflexión personal. Nuestro trabajo es brindarle tranquilidad e información respecto a que esperar en cada momento. Sin sorpresas. Esto hace una diferencia muy importante y facilita todo el proceso."

Estoy de acuerdo porque para realizarse una cirugía deben de pensarlo muy bien, ya que no siempre una operación al 100\% sale bien.

Diagnóstico

Según (Vásquez, 2015) "Mejorando el Contorno facial con un Aumento de Mentón en base al uso de Implantes: El crecimiento de la popularidad de intervenciones para el aumento de mentón con implantes se relaciona con el hecho de que esta parte del rostro determina en mucho la apariencia facial y si bien muchas personas no lo tienen claro, es un factor de equilibrio visual muy importante."

Para conseguir éxito en este tipo de cirugía, hay que conocer bien la estructura facial y sus características, para así poder equilibrar los elementos no armónicos. 


\section{Mentoplastia medio terapéutico y estético}

Vol. 1, núm. 5., (2017)

Vintimilla Burgos Napoleón Patricio; Plúas Robles Cecilia Isabel; Piña Tornés Arlines A.; Lenin Stalin Suasnabas Pacheco

Según (José Arruti, Álvaro Garcia-Rosado, Miguel Burgueño, 2012) para el diagnóstico de las deformaciones de mentón es necesario el Análisis estético facial. Estudios radiológicos: Rx. Panorámica de los maxilares, Teleradiografía de perfil, Teleradiografía frontal,Cefalometrías - Estudio de SAOS Polisomnografía nocturna, Cefalometrías: PAS (distancia de la base de la lengua a la pared posterior de la faringe), Fibroscopia: Maniobra de Müller (Mentoplastias, pág. 395)

Para el análisis facial son necesarios y obligatorios los exámenes complementarios, como las radiografías y fotografías; pues nos permiten diagnosticar el tipo de malformación o si el hueso del mentón es óptimo para el injerto.

Técnica Quirúrgica

La mentoplastía se puede realizar sola o combinarla con otras cirugías estéticas como la rinoplastia si se quiere llegar a una Perfiloplastía, asimismo es posible asociarla a Cirugía Ortognática.

Según (Espinoza, 2015) "La armonía en el rostro de ciertos pacientes se lo puede obtener con una simple modificación de la posición del mentón”,

En la cirugía del mentón también son utilizados pequeños implantes los cuales son diseñados para aumentar la estructura física del mentón y con ello tener una armonía facial perfecta. 


\section{Mentoplastia medio terapéutico y estético}

Vol. 1, núm. 5., (2017)

Vintimilla Burgos Napoleón Patricio; Plúas Robles Cecilia Isabel; Piña Tornés Arlines A.; Lenin Stalin Suasnabas Pacheco

\section{Según (Rodríguez, 2013) “Los implantes faciales están especialmente}

formados de materiales sólidos de silicona biocompatible,"

Esta cirugía es realizada en el hueso del mentón el cual se procede a mover depende de la posición que el cirujano a determinado para que el contorno facial sea armonioso.

Según (Estrella, 2012) 'La Mentoplastía es la cirugía utilizada para las modificaciones de la forma, tamaño o posición del mentón",

En la actualidad se han realizado estudios de que el mentón también produce lo que son los ronquidos es por ello que esta cirugía es realizada no solo por estética sino por una anomalía de la persona.

La osteosíntesis como medio de fijación del implante se realiza mediante miniplacas de titanio, mediante placas preformadas según el movimiento que se quiera realizar, y mediante tornillos bicorticales. Y procedimientos quirúrgicos de tejidos blandos que serán realizados conjuntamente con el cirujano plástico.

Según (Cali) "La mayoría de los pacientes describen el período de recuperación de una mentoplastía bastante fácil. La inflamación se observara más el tercer día después de la cirugía, y por lo general disminuye una semana después de la cirugía. La siguiente información le dará una idea de qué tipo de actividades son seguras durante el período de recuperación” 


\section{Mentoplastia medio terapéutico y estético}

Vol. 1, núm. 5., (2017)

Vintimilla Burgos Napoleón Patricio; Plúas Robles Cecilia Isabel; Piña Tornés Arlines A.; Lenin Stalin Suasnabas Pacheco

Efectivamente la cirugía del mentón no es tan dura como otras cirugías, pero esto no quita a que pueda a ver algún fallo durante la operación como eh dicho en otros artículos.

\section{Complicaciones y riesgos}

Según (Suñol, 2012). Cualquier tipo de actividad extraña es un riesgo inherente. La decisión individual de someterse a una intervención quirúrgica se basa en la comparación del riesgo con el beneficio potencial. Aunque la mayoría de los pacientes no experimentan las siguientes complicaciones, usted debería discutir cada una de ellas con su cirujano plástico para asegurarse de que comprende los riesgos, complicaciones potenciales y consecuencias de la mentoplastía (Cirugía estetica, 2012, pág. 1)

Es importante mencionar los riesgos y complicaciones de la mentoplastía y hacer obligatoriamente el consentimiento informado ya que a través de este el paciente tendrá el conocimiento necesario de lo que se va hacer en él.

Según Doris Rosario Betancourt Tinoco; Edwin Ernesto Vélez Leiva, 2009 "pueden haber complicaciones menores como; equimosis, hematoma, edemas, alergia, dehiscencia, desplazamiento, parestesia del labio inferior. Y mayores como; infección, incompetencia oral, desplazamiento, erosión ósea”(págs. 25-29).

Los riesgos y complicaciones menores que presenta cada paciente son debido a la poca información dada por el mismo durante una anamnesis, como las enfermedades que padece, el 


\section{Mentoplastia medio terapéutico y estético}

Vol. 1, núm. 5., (2017)

Vintimilla Burgos Napoleón Patricio; Plúas Robles Cecilia Isabel; Piña Tornés Arlines A.; Lenin Stalin Suasnabas Pacheco

uso de medicamentos, en cambio las mayores se deben a la falta de interconsultas que se deben mandar al paciente con otros especialistas así como los exámenes complementarios.

Según (Lupa karla; Mg.Sc Dr Bustamante Gladys, 2014)’La técnica anestésica con probabilidad de alergias, la colocación de implantes, se relaciona generalmente al uso de placas de titanio, con poca probabilidad de rechazo, pero la colocación irregular de las mismascondicionará a irregularidades de la zona que presentaran deformaciones y aspecto desagradable en la zona del procedimiento al igual que la sutura puede dejar una mala cicatrización”. (Riesgo de las Cirugías Mandibulares, pág. 2572)

Las complicaciones mentoplastía son escazas pues dependen en gran medida del mal diagnostica y la no realización de exámenes complementarios o a su vez el mal empleo de la técnica quirúrgica.

\section{Discusión.}

Si bien es cierto la metodología y la bibliografía nos permiten tener conocimiento sobre el tema de esté artículo y nos permite saber qué es un cirugía de mentón y para que nos sirve y demostrar así cuales son medios a usar en los diferentes, pero lo que está en discusión son los posibles riesgos de dicha cirugía; la Mentoplastía o Genioplastía en muchos de los casos no presenta ninguna complicación según lo expuesto por esos autores, pero al ser una acción quirúrgica debemos tomar en cuenta y tener bien presente que se debe considerar como riesgosa 


\section{Mentoplastia medio terapéutico y estético}

Vol. 1, núm. 5., (2017)

Vintimilla Burgos Napoleón Patricio; Plúas Robles Cecilia Isabel; Piña Tornés Arlines A.;

Lenin Stalin Suasnabas Pacheco

y sobre todo hay que tener un respaldo, como lo es el consentimiento informado; en el cual indicaremos al paciente todo acerca de la mentoplastía.

\section{Conclusiones.}

Como conclusiones podemos decir:

-La Mentoplastía es la cirugía de elección para la corrección de deformaciones de mentón que implican a su vez anomalías dentofaciales;

- El mentón es una fuente para injerto óseo autólogo para implantología dental,

-La Mentoplastía es una cirugía complementaria que devuelve al paciente la armonía en las estructuras dentofaciales.

\section{Bibliografía}

Burgoa, C. (2012). Mentoplastía. Actualización clínica investiga sobre la mentoplastía en Bolivia.

Cali, B. (s.f.). Operacion del ménton ( Mentoplastía). Aumento del Ménton reducir mentón, 2011.

Colmenero, C. (2012). Mentoplastia. Cirugía estética del mentón aislada o en combinación con cirugía ortognática en Uruguay.

Doris Rosario Betancourt Tinoco; Edwin Ernesto Vélez Leiva. (2009). MENTOPLASTIA DE AUMENTO CON IMPLANTE DE SILICONA . Obtenido de BIBLIOTECA DIGITAL DE LA UNIVERSIDAD CES: http://bdigital.ces.edu.co:8080/repositorio/bitstream/10946/1162/2/MENTOPLASTIA_A UMENTO_IMPLANTE_SILICONA.pdf

Espinoza, D. M. (2015). Mentoplastia. Cirugía Plástica de la Dra. Monica E.

Estrella, D. N. (2012). Mentoplastía. Cirugía Plastica .

Fernando Ramirez; Alejandra Buñuelos. (2006). Medigraphic, 65. 


\section{Mentoplastia medio terapéutico y estético}

Vol. 1, núm. 5., (2017)

Vintimilla Burgos Napoleón Patricio; Plúas Robles Cecilia Isabel; Piña Tornés Arlines A.; Lenin Stalin Suasnabas Pacheco

Freschi, A. (2011). Mentoplastia.

Gacitúa Garstman, H. (2012). Mentoplastía.

Gilbert, D. D. (2013). Mentoplastia (Aumento de Mentón). Dr Daniel Blum.

José Arruti, Álvaro Garcia-Rosado, Miguel Burgueño. (2012). Mentoplastias. Protocolos Clínicos de la Sociedad Española de Cirugía Oral Y Maxilofacial, 395.

L. Testut; O. Jacob. (s.f.). Tratado de Anatomia Topográfica. España: Salvat Editores S.A.

Laura García; Arturo Bilbao; Juan Grau. (s.f.). Injertos óseos en Implantología. Protocolos clinicos de la sociedad Española de Cirugia Oral y Maxilofacial , 151.

Lupa karla; Mg.Sc Dr Bustamante Gladys. (2014). Riesgo de las Cirugías Mandibulares. Actualización Clínica , 2572.

Macías, W. (03 de 12 de 2013). La Cirugía Plástica. Diario del Tiempo.

Marcela, M. P. (2014). Mentoplastia . Actualización Clínica Investiga, 5.

Mollinedo Patzi, M. (2011). Mentoplastía. Actualización clínica.

Morera, E. (s.f.). Tratamiento de las deformaciones del mentón. ACTA DE OTORRINOLARINGOL, (pág. 350). ESPAÑA.

Pazmiño, D. G. (2014). Mentoplastia. Medicos Ecuador.

Perez, R. (2011). Mentoplastia o cirugía del mentón.

Reyes Espinosa, J. A. (2012). Mejorando la armonía del rostro.

Rodríguez, D. N. (2013). Cirugía de Mentón. Cirugía Plasticas .

Ruiz, A. (2014). Alteración del mentón en Venezuela. Cirugía plástica de moda alteración del ménton.

Solano, D. G. (2013). Cirugía de aumento del mentón. Cirugia Plastica .

Suñol, J. (2012). Cirugía estetica. Consentimiento informado de la mentoplastía. Obtenido de https://drsunol.com/pdf/ci-mentoplastia-cirugia-estetica-joaquim-sunol.pdf

Valdivieso, D. J. (2012). Cirugía de Mentoplastía. Cirugías.

Vásquez, E. (2015). Aumento de mentón con implante. Cirugía plástica y estetica. 